\title{
Reducing triangular systems of ODEs with rational coefficients, with applications to coupled Regge-Wheeler equations
}

\author{
Igor Khavkine \\ khavkine@math.cas.cz \\ Institute of Mathematics, Czech Academy of Sciences, \\ Žitná 25, 11567 Praha 1, Czech Republic
}

\begin{abstract}
We concisely summarize a method of finding all rational solutions to an inhomogeneous rational ODE system of arbitrary order (but solvable for its highest order terms) by converting it into a finite dimensional linear algebra problem. This method is then used to solve the problem of conclusively deciding when certain rational ODE systems in upper triangular form can or cannot be reduced to diagonal form by differential operators with rational coefficients. As specific examples, we consider systems of coupled Regge-Wheeler equations, which have naturally appeared in previous work on vector and tensor perturbations on the Schwarzschild black hole spacetime. Our systematic approach reproduces and complements identities that have been previously found by trial and error methods.
\end{abstract}

\section{Introduction}

In the recent work [7], we have shown how, after a separation of variables, the radial mode equations of the vector wave equation $\square v_{\mu}=0$ on the Schwarzschild black hole spacetime may be significantly simplified by systematically decoupling them into an upper triangular form, where the diagonal components are generalized Regge-Wheeler operators and only a few of the off-diagonal components are non-vanishing. The original radial mode equations constitute a $4 \times 4$ second order linear ODE, whose components are coupled in a rather complicated way. The Regge-Wheeler operators appearing on the diagonal of the upper triangular form are second order scalar differential operators, with a well-studied spectral theory. This simplification makes it possible to transfer that knowledge to the study of the spectral theory of the original radial mode equations, which was otherwise rather unapproachable.

What is remarkable is that the original equations, the simplified upper triangular form, as well as the decoupling transformation are all ordinary differential operators with rational coefficients (at least in the standard Schwarzschild radial coordinate $r$ ). The existence of such such a simplification, in particular the ability to set to zero most of the off-diagonal terms in the upper triangular form, follows from specific identities previously discovered by trial and error in [6], and in part also in [8] (see [7] for a full discussion), and is certainly not obvious a priori. This naturally raises the questions of how these identities could be recovered in a systematic approach, and whether more could be discovered to push the simplifications described above as far as possible. These questions become particularly relevant for trying to repeat the same simplifications for the Lichnerowicz equation $\square p_{\mu \nu}-2 R_{(\mu}{ }^{\lambda \kappa}{ }_{\nu)} p_{\lambda \kappa}=0$, which has a role relative to linearized Einstein equations analogous to that of the vector wave equation relative to Maxwell equations. The relevant identities were also discovered in [6], but only by means of voluminous trial and error calculations and without a clear answer to whether they could be further improved. We will revisit this point when considering examples in Section 4.1.

The above questions were left open in [7] and are answered in this work. The main systematic tool at our disposal is the theory of rational solutions of ordinary differential equations (ODEs) with rational coefficients. Under appropriate mild hypotheses on the equation, the search for such solutions can be reduced to a finite dimensional linear algebra problem (Theorem 2.4). We summarize this theory in Section 2. The theory of rational solutions of scalar rational ODEs is fairly well developed (cf. the monograph [3] and the references therein; more precise references are given in the text). Our innovation is to synthesize this approach into an economical form, based on what we call leading (or trailing) multipliers, that is directly applicable to our examples of interest, but also more generally to ODE systems of arbitrary size and order. In Section 3, we consider the problem of setting to zero an off-diagonal block in an upper triangular rational ODE system by a transformation with rational coefficients. This problem is first reduced to an operator identity (Equation (15)), which in turn can be solved by converting it into 
an inhomogeneous rational ODE system (Theorem 3.1). Finally, in Section 4, we combine the results of Sections 2 and 3 to show how the special identities used in [7] and [6] can be recovered with minimal effort, especially when aided by computer algebra. In particular, we can conclusively decide when simplifications of the kind described earlier do or do not exist, with several examples given in Section 4.1. Section 5 concludes with a discussion of the results and an outlook to further work.

\section{Rational solutions of ODEs with rational coefficients}

The main objects under our study will be ordinary differential operators and equations (ODEs) with rational coefficients. We will usually denote the independent variable by $r$. A differential operator $e$ applied to a function $u=u(r)$ will be denoted by $e[u]$. Both $u$ and $e[u]$ could be vector valued. We do not put any a priori bounds on the dimensions or differential order of $e$. Hence, $e$ can also be seen as a matrix of scalar differential operators. Hence, when $e$ is of order zero, $e[u]$ will correspond to multiplication of $u$ by an $r$-dependent matrix. We will denote the composition of differential operators by $\circ$, so that $e \circ f[u]=e[f[u]]$. We will restrict our attention only to differential operators with rational coefficients and in general complex valued.

In this section, we will eventually show how to find all the rational solutions $u=u(r)$ of a rational ODE $e[u]=v$, by reducing it to a finite dimensional linear algebra problem. Our approach starts with a Laurent series representation $u=\sum_{n} u_{n} r^{n}$ converts the equation $e[u]=v$ into a recurrence relation on the coefficients of $u$. At different stages, it would be useful to consider Laurent series of different kinds. In particular, we will mostly deal with formal series (no requirement of convergence). However, convergence will be automatic if we know in advance that the series has only finitely many terms or that it comes from the expansion of a rational function. Thus, we may distinguish unbounded Laurent series $\mathbb{C}\left[\left[r, r^{-1}\right]\right]$, bounded (from below) Laurent series $\mathbb{C}\left[r^{-1}\right][[r]]$, bounded from above Laurent series $\mathbb{C}[r]\left[\left[r^{-1}\right]\right]$ and Laurent polynomials $\mathbb{C}\left[r, r^{-1}\right]$. Of course, we could also consider Laurent series centered at some other $r=\rho \neq 0$, but for convenience of notation whenever possible we will stick with $\rho=0$.

For bounded (from below) Laurent series, it is helpful to define leading or trailing orders and coefficients. For $u=\sum_{n} u_{n} r^{n} \in \mathbb{C}\left[r^{-1}\right][[r]]$, if we can write

$$
u=\left[\begin{array}{c}
u_{n_{1}}^{1} r^{n_{1}}(1+O(r)) \\
u_{n_{2}}^{2} r^{n_{2}}(1+O(r)) \\
\vdots
\end{array}\right],
$$

with each $O(r) \in r \mathbb{C}[[r]]$, the $n_{1}, n_{2}, \ldots$ are the leading orders of the components $u^{1}, u^{2}, \ldots$ of $u$, with the exception when $u_{n_{i}}^{i}=0$, in which case we set $n_{i}=+\infty$. We denote by $|\check{u}|$ the vector where each component of $u$ is replaced by its leading order, and we refer to it as the leading order of $u$. When $n=\min _{i} n_{i}<\infty, u_{n} \neq 0$ and we call it the leading coefficient of $u$. We define the leading coefficient of 0 to be 0 .

Similarly, for bounded from above Laurent series $u \in C[r]\left[\left[r^{-1}\right]\right]$, if we can write

$$
u=\left[\begin{array}{c}
u_{n_{1}}^{1} r^{n_{1}}\left(1+O\left(r^{-1}\right)\right) \\
u_{n_{2}}^{2} r^{n_{2}}\left(1+O\left(r^{-1}\right)\right) \\
\vdots
\end{array}\right],
$$

with each $O\left(r^{-1}\right) \in r^{-1} \mathbb{C}\left[\left[r^{-1}\right]\right]$, the $n_{1}, n_{2}, \ldots$ are the trailing orders of the components $u^{1}, u^{2}, \ldots$ of $u$, with the exception when $u_{n_{i}}^{i}=0$, in which case we set $n_{i}=-\infty$. The trailing order $|\hat{u}|$ of $u$ is the vector of the trailing orders of the components of $u$. When $n=\max _{i} n_{i}>-\infty, u_{n} \neq 0$ and we call it the trailing coefficient of $u$. We define the trailing coefficient of 0 to be 0 .

Clearly the leading (trailing) coefficient of a bounded (from above) Laurent series vanishes if and only if the whole series vanishes. For Laurent polynomials $u \in \mathbb{C}\left[r, r^{-1}\right]$, both the leading and trailing orders, and coefficients, are well-defined.

Consider an ODE $e[u]=0$ on bounded Laurent series $u=\sum_{n} u_{n} r^{n} \in \mathbb{C}\left[r^{-1}\right][[r]]$. What we like to do is turn $e[u]=0$ into a linear recurrence relation on the coefficients $u_{n}$ of the form $E_{n} u_{n}=f_{n}\left(u_{n-1}, u_{n-2}, \ldots\right)$ and then uniquely solve for $u_{n}$ as a function of $u_{n-1}$ and lower order coefficients, for almost all $n$ (that is, all but finitely many). Those finitely many $n$ for which the solution for $u_{n}$ would not be unique would then determine the dimension of the solution space of the ODE. This approach requires that the coefficients $E_{n}$ in the recurrence relation be invertible for almost all $n$. For scalar equations this is an almost trivial requirement, but in matrix equations different components of $e$ may be weighted so differently by powers of $r$ that the coefficient $E_{n}$ comes out 
as a singular matrix for infinitely many $n$. Often this problem can be remedied by applying suitable transformations to $u$ and to $e[u]$.

Let $S=S(r)$ and $T=T(r)$ be matrices with Laurent polynomial components. For future convenience, we also require that the inverses $S^{-1}$ and $T^{-1}$ also have Laurent polynomial components (or, equivalently, the determinants of $S$ and $T$ are proportional to single powers of $r$ ). We say that $S$ and $T$ are respectively the source and target leading multipliers of $e$ when, after expanding all rational coefficients as bounded Laurent series, we have

$$
e\left[S(r) u_{n} r^{n}\right]=T(r)\left(E_{n} u_{n} r^{n}+r^{n} O(r)\right),
$$

with the components of $O(r)$ all in $r \mathbb{C}[[r]]$ and $E_{n}$ an $r$-independent matrix that is invertible for almost all $n$. We call $E_{n}$ the trailing characteristic matrix of $e$ with respect to the given multipliers. Similarly, we say that $S$ and $T$ are respectively the source and target trailing multipliers of $e$ when, after expanding all rational coefficients as bounded from above Laurent series, we have

$$
e\left[S(r) u_{n} r^{n}\right]=T(r)\left(E_{n} u_{n} r^{n}+r^{n} O\left(r^{-1}\right)\right),
$$

with the components of $O\left(r^{-1}\right)$ all in $r^{-1} \mathbb{C}\left[\left[r^{-1}\right]\right]$ and $E_{n}$ an $r$-independent matrix that is invertible for almost all $n$. We call $E_{n}$ the trailing characteristic matrix of $e$ with respect to the given multipliers.

Those integer $n \in \mathbb{Z}$ such that $\operatorname{det} E_{n}=0$, which is a polynomial in $n$, are called (respectively leading or trailing) (integer) characteristic roots or exponents of $e$ with respect to given multipliers $S, T$. We denote the set of such leading characteristic exponents by $\check{\sigma}(e)$ and the set of such trailing characteristic exponents by $\hat{\sigma}(e)$, with implicit dependence on the $S, T$ multipliers, of course.

We will not dwell on when leading or trailing multipliers exist, but will just assume that they are given for any particular problem. Often $S$ and $T$ may be taken to be diagonal, with appropriately chosen powers of $r$ on the diagonal. Otherwise, they could be determined by a recursive procedure similar to that used in the analysis of regular and irregular singularities for ODEs with meromorphic coefficients [11].

Any rational $u \in \mathbb{C}(r)$ will have a (convergent) bounded Laurent series expansion about any point $r=\rho$. Without loss of generality, let us take $\rho=0$. We would like to prove some bounds on the leading order of $u$ at $r=0$ when it solves $e[u]=v$, with some rational $v \in \mathbb{C}(r)$. For this purpose, it is actually more natural to allow $u$ and $v$ to be bounded Laurent series.

Lemma 2.1. Let $e[u]=0$ be an $O D E$ with rational coefficients, leading multipliers $S, T$, and leading characteristic matrix $E_{n}$. Let $u, v \in \mathbb{C}\left[r^{-1}\right][[r]]$ with leading orders $m=\min _{i}\left|\check{u}^{i}\right|, n=\min _{i}\left|\check{v}^{i}\right|$ (the values $m=\infty$ or $n=\infty$ are both permissible). If $e[S u]=T v$, then either (a) $m=n$ and (provided $n<\infty$ ) $v_{n}=E_{n} u_{n}$ or (b) $m$ is a leading characteristic exponent of $e, E_{m} u_{m}=0$, and $m<n$. In other words

$$
\min \{n\} \cup \check{\sigma}(e) \leq m \text {. }
$$

This result and proof are analogous to those presented in $\S 6$ of [3], where only the case of scalar equations and polynomial coefficients is treated. The monograph [3] cites $[2,1,5]$ as the original sources for the basic ingredients of the approach. A generalization of the approach to systems of arbitrary size and order can be found in [4] (which also cites slightly earlier related work). Our innovation is to synthesize this approach into an economic method, as presented in this section, based on the convenient notion of leading (trailing) multipliers $S, T$ and the way they lead to the leading (trailing) characteristic matrix $E_{n}$.

Proof. If $m=\infty$, this means that $u=0$. Then also $v=0$ and $n=\infty$, meaning that (a) holds. For the rest we will assume that $m<\infty$, meaning that $u$ has the non-vanishing leading coefficient $u_{m} \neq 0$. If $E_{m} u_{m} \neq 0$, then the defining property (3) of leading multipliers $S$ and $T$ directly implies part (a), that is $n=m$. On the other hand, if $E_{m} u_{m}=0$ and since by definition $u_{m} \neq 0$, the leading order of $u$ must be a characteristic exponent of $e, m \in \check{\sigma}(e)$. Using again (3), we also find $m<n$.

Part (a) implies $n \leq m$, while part (b) implies $\min \check{\sigma}(e) \leq m$ and $m<n$. Since at least one of (a) or (b) always holds, the lower bound (5) on $m$ is always true.

All the same arguments apply to Laurent expansions about $r=\infty$, though after making use of the transformation $r \mapsto 1 / r$. For convenience, we state the corresponding result without the need to invoke this transformation.

Lemma 2.2. Let $e[u]=0$ be an $O D E$ with rational coefficients, trailing multipliers $S, T$, and trailing characteristic matrix $E_{n}$. Let $u, v \in \mathbb{C}[r]\left[\left[r^{-1}\right]\right]$ with trailing orders $m=\max _{i}\left|\hat{u}^{i}\right|, m=\max _{i}\left|\hat{v}^{i}\right|$ (the values $m=-\infty$ or $n=-\infty$ are both permissible). If $e[S u]=T v$, then either (a) $m=n$ and (provided $n>-\infty) v_{n}=E_{n} u_{n}$ or (b) $m$ is a trailing characteristic exponent of $e$ and $E_{m} u_{m}=0$. In other words

$$
m \leq \max \{n\} \cup \hat{\sigma}(e) .
$$


Now we know how to bound the order of the pole of a rational solution $u$ at any particular value of $r=\rho \in \mathbb{C}$. For the following class of ODEs, we can also identify all the potential locations of the poles of $u$.

Lemma 2.3. Let $e[u]=0$ be an ODE of differential order $p$ with rational coefficients, for which there exists an invertible matrix $P=P(r)$ with rational coefficients such that $P e[u]=\frac{d^{p}}{d r^{p}} u+\tilde{e}[u]$, where $\tilde{e}$ is of differential order at most $p-1$. For rational $u, v \in \mathbb{C}(r)$, if $e[u]=v$, then $u(r)$ is smooth (i.e., has no pole) at all but finitely many points of $\mathbb{C}$. The only possible exceptions are $r=\rho$, with $\rho$ one of the poles of P $v$ or of the coefficients of $\tilde{e}$.

Proof. By our hypotheses, we can put the equation $e[u]=v$ into the equivalent form

$$
\frac{d^{p}}{d r^{p}} u+\tilde{e}[u]=P v
$$

where $P v$ is rational and $\tilde{e}[u]$ has rational coefficients. Consider a point $r=\rho \in \mathbb{C}$, that is not pole of $P v$ or of the coefficients of $\tilde{e}[u]$. There are obviously only finitely many such excluded points. If $u$ has a pole of type $(r-\rho)-k$, then $\frac{d^{p}}{d r^{p}} u$ has a pole of type $(r-\rho)^{-k-p}$, while $P v$ and $\tilde{e}[u]$ will only have poles of lower order. But this means that such a $u$ cannot be a solution of our equation. Hence, any rational solution $u \in \mathbb{C}(r)$ can have poles only in the already mentioned excluded set.

Given a rational ODE $e[u]=0$, when considering Laurent expansions at $r=\rho$, let us denote the corresponding leading multipliers by $S_{\rho}, T_{\rho}$, which are by definition rational and have poles only at $r=\rho$ (and $r=\infty$, of course). For a rational $u \in \mathbb{C}(r)$, if we know that its poles are restricted to a finite set of points in $\mathbb{C}$ and we have a bound on the degree of the pole at each of these points, then we can find a rational matrix $R=R(r)$ such that $R u$ has no poles in $\mathbb{C}$.

Theorem 2.4. Let $e[u]=v$ be an $O D E$ with rational coefficients and rational $v \in \mathbb{C}(r)$, satisfying the hypotheses of Lemma 2.3. Suppose also that we have the leading multipliers $S_{\rho}, T_{\rho}$ of e at each of the finitely many exceptional points $r=\rho \in \mathbb{C}$ identified in Lemma 2.3. Then, there exists a rational matrix $R=R(r)$ such that, for any rational $u \in \mathbb{C}(r)$ satisfying $e[u]=v$, there is a Laurent polynomial $\tilde{u} \in \mathbb{C}\left[r, r^{-1}\right]$ satisfying $u=R \tilde{u}$.

We call such a matrix $R$ a universal multiplier for the rational inhomogeneous ODE $e[u]=v$. A universal multiplier certainly need not be unique. The existence of universal multipliers for scalar equations is discussed in $[3, \S 7]$, which cites $[1,5]$ as original references. For systems of arbitrary size and order, the existence of universal multipliers is discussed for instance in [4] (with references to slightly earlier work).

Proof. A rational $u \in \mathbb{C}(r)$ has only finitely many poles, and at each of those poles it has a bounded Laurent series expansion. By invoking Lemma 2.3 we can constrain the poles of $u$ to a finite set of points. Then, by invoking Lemma 2.1, for each of those points, say $r=\rho$, we can find a finite lower bound $\check{n}_{\rho}$ for the leading Laurent order of $S_{\rho}^{-1} u$ at $r=\rho$. Recall that one of the defining properties of $S_{\rho}$ is that both it and $S_{\rho}^{-1}$ only have poles at $r=\rho$ (and of course at $r=\infty$ ). This means that $\tilde{u}=\prod_{\rho} S_{\rho}^{-1}(r-\rho)^{-\tilde{n}_{\rho}} u$, where the product is taken over the potential pole locations (possibly excluding $\rho=0$ ), is still rational but no longer has any poles in $r \in \mathbb{C}$, with the possible exception of $r=0$. But that can only be if $\tilde{u} \in \mathbb{C}\left[r, r^{-1}\right]$ is a Laurent polynomial. Therefore, we can take

$$
R(r)=\prod_{\rho} S_{\rho}(r)(r-\rho)^{\check{n}_{\rho}}
$$

as the desired universal multiplier. Since any of the $\check{n}_{\rho}$ can be decreased without breaking this result, we have many possible choices for $R$.

Corollary 2.5. Let $e$ and $v$ be as in Theorem 2.4, with universal multiplier $R$. In addition, suppose that we have the leading multipliers $S_{0}, T_{0}$ at $r=0$ and the trailing multipliers $S_{\infty}, T_{\infty}$ at $r=\infty$ for $\tilde{e}=e \circ R$. Then the equation $e[u]=v$ for $u$ can be reduced to a finite dimensional linear system, and hence its solution space is finite dimensional.

Proof. By invoking Theorem 2.4, solving $e[u]=v$ for $u \in \mathbb{C}(r)$ is equivalent to solving $\tilde{e}[\tilde{u}]=v$ for $\tilde{u} \in \mathbb{C}\left[r, r^{-1}\right]$, with $u=R \tilde{u}$ and $\tilde{e}[\tilde{u}]=e[R u]$. Invoking Lemma 2.1 we can find a finite lower bound on the leading order of $S_{0}^{-1} \tilde{u}$ and hence of $\tilde{u}$, which we will call $\check{n}$. Invoking Lemma 2.2 we can find a finite upper bound on the leading order of $S_{\infty}^{-1} \tilde{u}$ and hence of $\tilde{u}$, which we will call $\hat{n}$. Therefore, we can parametrize all solutions as Laurent polynomials

$$
\tilde{u}=\sum_{n=\check{n}}^{\hat{n}} u_{n} r^{n},
$$


which has $\hat{n}-\check{n}+1<\infty$ undetermined coefficients. Plugging this parametrization into the equation $\tilde{e}[\tilde{u}]=v$, putting both sides over a common denominator, and comparing coefficients reduces the original problem to a finite dimensional linear system of equations. The dimension of the solution space of this system is of course finite, and (crudely) bounded by the number of coefficients in (9).

Of course, once an equation has been reduced to an explicit finite dimensional linear system, it can be solved on a computer, even symbolically.

\section{Reducing triangular ODE systems with rational coefficients}

In this section, we are interested in the following question. Given an ODE system in block upper triangular form, is it possible to find an equivalent ODE system where the off-diagonal block has been set to zero, hence in diagonal form? If possible, we call this a reduction to block diagonal form and say that the original system can be reduced. A refined version of the question is whether a rational ODE system can be reduced while remaining rational.

The first thing we need to clarify is the notion of equivalence. Roughly speaking, two ODE systems should be equivalent when there is an isomorphism between their solution spaces. A further practical requirement is that this isomorphism be given, in either direction, by differential operators. After all, transformations given by differential operators tend to be easier to write down in terms of explicit formulas, while also allowing rather precise control over some properties of the coefficients of the ODE systems, like rationality or upper triangular form. Also, an equivalence should make explicit the transformation of one ODE system into the other one, again by a differential operator.

We formalize these ideas as follows. Given two ODE systems, $e[u]=0$ and $\bar{e}[\bar{u}]=0$, an equivalence between them consists of pairs of differential operators $k, g$ and $\bar{k}, \bar{g}$ obeying the operator identities, for any $u, \bar{u}$ and $v, \bar{v}$,

$$
\begin{array}{rlrl}
\bar{e}[k[u]] & =g[e[u]], & \bar{k}[k[u]] & =u, \\
e[\bar{k}[\bar{u}]] & =\bar{g}[\bar{e}[\bar{u}]], & & \bar{g}[g[v]]=v, \\
k[\bar{k}[\bar{u}]]=\bar{u}, & & g[\bar{g}[\bar{v}]]=\bar{v} .
\end{array}
$$

Graphically, if we represent each differential operator by an arrow and appropriate function spaces by •'s, these identities mean that the squares in the following diagram are commutative and that the horizontal arrows compose to identity in either direction:

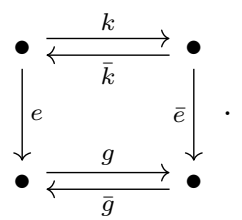

Basically, these identities imply that for a solution $u$ of $e[u]=0, \bar{u}=k[u]$ is a solution of $\bar{e}[\bar{u}]=0$, and vice versa, where the barred and unbarred transformation operators are mutually inverse. Finally, when dealing with ODE systems with rational coefficients, we require the coefficients of the operators $k, g$ and $\bar{k}, \bar{g}$ to be rational as well.

The above notion of equivalence is actually somewhat more rigid than absolutely necessary, but it will be sufficient for our purposes. A discussion of a somewhat looser notion of equivalence can be found in [7], with references to deeper literature on this topic. Below, we use this notion of equivalence to discuss reduction of triangular ODE systems. A similar discussion can already be found in [7, Sec.2.3].

An ODE system of the form

$$
\left[\begin{array}{cc}
e_{0} & \Delta \\
0 & e_{1}
\end{array}\right]\left[\begin{array}{l}
u_{0} \\
u_{1}
\end{array}\right]=\left[\begin{array}{l}
0 \\
0
\end{array}\right]
$$

is said to be (block) upper triangular, or (block) diagonal if $\Delta=0$. We will always assume that this system has rational coefficients. We presume also that the equations $e_{0}\left[u_{0}\right]=0$ and $e_{1}\left[u_{1}\right]=0$ are ODE systems of unspecified dimensions and differential orders, but such that they can be solved for the highest derivatives, as in the hypotheses of Lemma 2.3.

A reduction of the upper triangular ODE system (13) is an equivalence given by the following pair of commutative 
diagrams

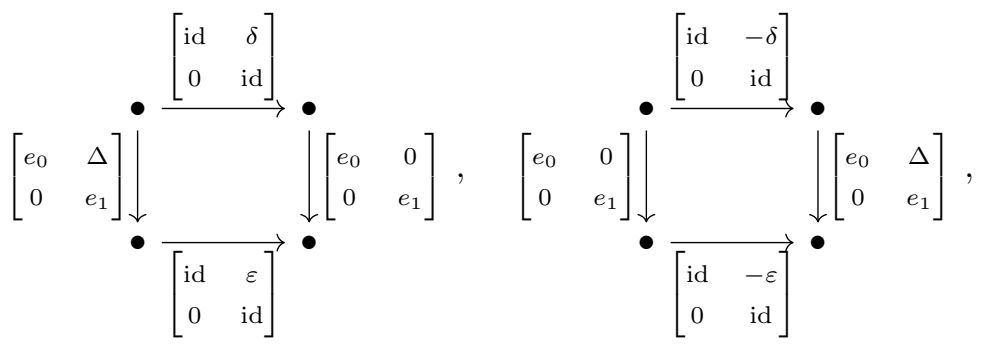

where the corresponding horizontal arrows are clearly mutual inverses. Of course, we require the differential operators $\delta$ and $\varepsilon$ to have rational coefficients. By direct calculation, we can check that the above diagrams commute if and only if $\delta$ and $\varepsilon$ satisfy the operator identity

$$
e_{0} \circ \delta=\Delta+\varepsilon \circ e_{1} .
$$

Note that solutions of (15) are certainly not unique. For instance, for any $\delta, \varepsilon$ solution pair, $\left(\delta+\alpha \circ e_{1}\right),\left(\varepsilon+e_{0} \circ \alpha\right)$ is another solution, with arbitrary $\alpha$, since $e_{0} \circ\left(\alpha \circ e_{1}\right)=\left(e_{0} \circ \alpha\right) \circ e_{1}$. In addition, having a solution pair $\delta, \varepsilon$ for a given $\Delta$, automatically gives us the solution pairs $(\delta-\alpha),(\varepsilon+\beta)$ for $\Delta$ replaced with $\Delta+e_{0} \circ \alpha+\beta \circ e_{1}$. When $e_{0}\left[u_{0}\right]=0$ and $e_{1}\left[u_{1}\right]=0$ can be solved for their highest derivatives, we can use the above freedom to reduce equation (15), with $\delta, \varepsilon$ and $\Delta$ of potentially high differential orders, to the same equation, but with the differential orders of $\delta, \varepsilon$ and $\Delta$ bounded by the orders of $e_{0}$ and $e_{1}$.

Theorem 3.1. Suppose that the rational ODE systems $e_{0}\left[u_{0}\right]=0$ and $e_{1}\left[u_{1}\right]=0$ of differential orders $p_{0}$ and $p_{1}$, respectively. Suppose also that they can be solved for the highest order derivatives, that is, for $i=0,1$ there exist rational invertible matrices $P_{i}$ such that $P_{i} e_{i}[u]=\frac{d^{p_{i}}}{d r^{p_{i}}} u+\tilde{e}_{i}[u]$, where $\tilde{e}_{i}$ is of differential order $<p_{i}$. (a) The knowledge of $\delta$ and $\Delta$ in (15) is sufficient to reconstruct $\varepsilon$ uniquely. (b) For given $\Delta$ and a $\delta$ of fixed differential order, the existence of an $\varepsilon$ satisfying (15) is equivalent to a rational ODE system on the coefficients of $\delta$. (c) If $\Delta$ if of differential order $<p_{0}+p_{1}$, then (15) has a solution if and only if it has a solution where $\delta$ is of differential order $<p_{1}$ and $\varepsilon$ is of differential order $<p_{0}$.

Proof. We first make the standard observation is that, under our hypotheses on $e_{i}(i=0,1)$, for any differential operator $f_{i}\left[u_{i}\right]$, we can find a unique differential operators $g_{i}$ and $\tilde{f}_{i}$ such that $f_{i}=g_{i}+\tilde{f}_{i} \circ e_{i}$, with $g_{i}$ of differential order $<p_{i}$. This is easy to prove by noting that we cannot decrease the differential order of $e_{i}$ by pre-composing it with a non-zero differential operator and then recursively rewriting the highest order derivatives in $f_{i}$, say $\frac{d^{p_{i}+q}}{d r^{p_{i}+q}} u_{i}$, as $-\frac{d^{q}}{d r^{q}} \tilde{e}_{i}[u]+\frac{d^{q}}{d r^{q}} P_{i} e_{i}\left[u_{i}\right]$. Obviously, both $g_{i}$ and $\tilde{f}_{i}$ also have rational coefficients and, in fact, their coefficients are linear rational differential operators applied to the coefficients of $f_{i}$.

To prove part (a), note that the identity $e_{0} \circ \delta-\Delta=0+\varepsilon \circ e_{1}$, combined with our initial observation, implies that $\varepsilon$ is uniquely fixed once we know $\Delta$ and $\delta$.

To prove part (b), consider the decomposition $e_{0} \circ \delta-\Delta=\tilde{\Delta}+\varepsilon \circ e_{1}$, with $\tilde{\Delta}$ of differential order $<p_{1}$, which by our initial observation always exists and is unique. Thus, $\delta, \varepsilon$ and $\Delta$ satisfy (15) if and only if the coefficients of $\tilde{\Delta}$ are all zero. But construction, the coefficients of $\tilde{\Delta}$ are linear rational differential operators acting on the coefficients of $\delta$ and $\Delta$.

To prove part (c), we first apply our initial observation to get the decomposition $\delta=\tilde{\delta}+\varepsilon_{1} \circ e_{1}$, where the differential order of $\tilde{\delta}$ is $<p_{1}$. Then $e_{0} \circ \tilde{\delta}=\Delta+\tilde{\varepsilon} \circ e_{1}$, with $\tilde{\varepsilon}=\varepsilon-\varepsilon_{1}$. The differential orders of $e_{0} \circ \tilde{\delta}$ and $\Delta$ are both $<p_{0}+p_{1}$, hence by comparison we can conclude that the differential order of $\tilde{\varepsilon}$ is $<p_{0}$.

\section{Systems of Regge-Wheeler equations}

Let

$$
f(r)=1-\frac{2 M}{r}, \quad f^{\prime}(r)=\frac{f_{1}(r)}{r}, \quad f_{1}(r)=\frac{2 M}{r} .
$$

Define the (generalized) spin-s Regge-Wheeler operator with mass parameter $M$, angular momentum quantum number $l$ and frequency $\omega$ by

$$
\mathcal{D}_{s} \phi=\partial_{r} f \partial_{r} \phi-\frac{1}{r^{2}}\left[\mathcal{B}_{l}+\left(1-s^{2}\right) f_{1}\right] \phi+\frac{\omega^{2}}{f} \phi,
$$

where $\mathcal{B}_{l}=l(l+1)$, with $l=0,1,2, \ldots$ We will assume that $\omega \neq 0$ and that $s$ is a non-negative integer. Of course, for any $s, \mathcal{D}_{s}$ has rational coefficients and satisfies the hypotheses of Lemma 2.3. 
Consider the upper triangular rational ODE system

$$
\left[\begin{array}{cc}
\mathcal{D}_{s_{0}} & \Delta \\
0 & \mathcal{D}_{s_{1}}
\end{array}\right]\left[\begin{array}{l}
u_{0} \\
u_{1}
\end{array}\right]=\left[\begin{array}{l}
0 \\
0
\end{array}\right]
$$

where we suppose that $\Delta$ is of differential order at most 1 . As discussed in Section 3, this system is reducible to diagonal by an equivalence (Section 3) with rational coefficients if and only if the following version of Equation 15 is satisfied:

$$
\mathcal{D}_{s_{0}} \circ \delta=\Delta+\varepsilon \circ \mathcal{D}_{s_{1}} .
$$

By Theorem 3.1, without loss of generality, we can consider this problem restricted to the following class of operators:

$$
\begin{aligned}
\Delta & =\frac{1}{r^{2}}\left(\Delta_{1} r \partial_{r}+\Delta_{0}\right), \\
\delta & =\delta_{1} r \partial_{r}+\delta_{0}, \\
\varepsilon & =\delta_{1} r \partial_{r}+\left[2 \partial_{r}\left(r \delta_{1}\right)-\frac{f_{1}}{f} \delta_{1}+\delta_{0}\right],
\end{aligned}
$$

where $\delta_{i}, \Delta_{i}$, for $i=0,1$, are all rational functions. Plugging this parametrization into (19) and comparing coefficients, we find the equivalent ODE system

$$
\begin{aligned}
& e\left[\begin{array}{l}
\delta_{0} \\
\delta_{1}
\end{array}\right]:=\left[\begin{array}{ll}
f & 0 \\
0 & f
\end{array}\right] r^{2} \partial_{r}^{2}\left[\begin{array}{l}
\delta_{0} \\
\delta_{1}
\end{array}\right]+\left[\begin{array}{cc}
f_{1} & -2 \frac{\omega^{2} r^{2}}{f}+2\left[\mathcal{B}_{l}+f_{1}\left(1-s_{1}^{2}\right)\right] \\
2 f & 2 f-f_{1}
\end{array}\right] r \partial_{r}\left[\begin{array}{l}
\delta_{0} \\
\delta_{1}
\end{array}\right] \\
& +\left[\begin{array}{cc}
f_{1}\left(s_{0}^{2}-s_{1}^{2}\right) & -2 \frac{\omega^{2} r^{2}\left(f-f_{1}\right)}{f^{2}}-\frac{f_{1}}{f}\left[\mathcal{B}_{l}+1-s_{1}^{2}\right] \\
0 & f_{1}\left(s_{0}^{2}-s_{1}^{2}+\frac{1}{f}\right)
\end{array}\right]\left[\begin{array}{c}
\delta_{0} \\
\delta_{1}
\end{array}\right]=\left[\begin{array}{c}
\Delta_{0} \\
\Delta_{1}
\end{array}\right]
\end{aligned}
$$

for $\delta_{0}, \delta_{1}$, with $\Delta_{0}, \Delta_{1}$ as inhomogeneous sources.

Next, we will apply the analysis of Section 2 to check the conditions under which the system (23) has rational solutions for $\delta_{0}, \delta_{1}$, with given $\Delta_{0}, \Delta_{1}$.

It is easy to see that the only singular points of the equation (23) are $r=0,2 M, \infty$ (recall that $f(2 M)=0$ ). In this work, we will not consider $\Delta_{0}, \Delta_{1}$ with poles at other values of $r$, which means that these points are the only possible locations of the poles of $\delta_{0}, \delta_{1}$.

For each of the singular points, we have the following multipliers $(S$ and $T)$, characteristic matrices $\left(E_{n}\right)$ and characteristic exponents $(\sigma(e))$.

- $r=0: \check{\sigma}_{0}(e)=\left\{ \pm s_{0} \pm s_{1}\right\}$, with $\operatorname{det} E_{n}=(2 M)^{2}\left(n+s_{0}+s_{1}\right)\left(n+s_{0}-s_{1}\right)\left(n-s_{0}+s_{1}\right)\left(n-s_{0}-s_{1}\right)$ and

$$
S=\left[\begin{array}{ll}
1 & 0 \\
0 & 1
\end{array}\right], \quad T=\left[\begin{array}{cc}
r^{-1} & 0 \\
0 & r^{-1}
\end{array}\right], \quad E_{n}=-2 M\left[\begin{array}{cc}
n^{2}-2 n-s_{0}^{2}+s_{1}^{2} & -2 n\left(1-s_{1}^{2}\right) \\
2 n & n^{2}+2 n-s_{0}^{2}+s_{1}^{2}
\end{array}\right] .
$$

- $r=2 M: \check{\sigma}_{2 M}(e)=\{-1\}$, with $\operatorname{det} E_{n}=(n+1)^{2}\left[(n+1)^{2}+16 M^{2} \omega^{2}\right]$ and

$$
S=\left[\begin{array}{cc}
\frac{(r-2 M)}{2 M} & 0 \\
0 & \frac{(r-2 M)^{2}}{4 M^{2}}
\end{array}\right], \quad T=\left[\begin{array}{cc}
1 & 0 \\
0 & \frac{2 M}{(r-2 M)}
\end{array}\right], \quad E_{n}=\left[\begin{array}{cc}
(n+1)^{2} & -8 M^{2} \omega^{2}(n+1) \\
2(n+1) & (n+1)^{2}
\end{array}\right] .
$$

- $r=\infty: \hat{\sigma}_{\infty}(e)=\{-1,0\}$, with $\operatorname{det} E_{n}=4 \omega^{2} n(n+1)$ and

$$
S=\left[\begin{array}{ll}
1 & 0 \\
0 & 1
\end{array}\right], \quad T=\left[\begin{array}{cc}
r^{2} & 0 \\
0 & 1
\end{array}\right], \quad E_{n}=\left[\begin{array}{cc}
0 & -2 \omega^{2}(n+1) \\
2 n & n(n+1)
\end{array}\right] .
$$

Suppose that $A_{\rho}=T_{\rho}^{-1}\left[\begin{array}{c}\Delta_{0} \\ \Delta_{1}\end{array}\right]$ has leading order $\check{m}=\min _{i}\left|\check{A}_{0}^{i}\right|$, and trailing order $\hat{m}=\max _{i}\left|\hat{A}_{\infty}^{i}\right|$. At $r=2 M$, we do not need the specific order, but just some lower bound $m \leq \min _{i}\left|\check{A}_{2 M}^{i}\right|_{r=2 M}$ for some integer $m \leq-1$. We choose a bound of this form because then the identity

$$
\min \{m\} \cup \check{\sigma}_{2 M}(e)=\min \{m,-1\}=m
$$


determines that the Laurent series expansion of the solution $\delta=\left[\begin{array}{l}\delta_{0} \\ \delta_{1}\end{array}\right]$ must belong to

$$
\delta \in\left[\begin{array}{cc}
\frac{(r-2 M)}{2 M} & 0 \\
0 & \frac{(r-2 M)^{2}}{4 M^{2}}
\end{array}\right](r-2 M)^{\min \{m\} \cup \check{\sigma}_{2 M}(e)} \mathbb{C}[[(r-2 M)]]=\left[\begin{array}{cc}
1 & 0 \\
0 & \frac{(r-2 M)}{2 M}
\end{array}\right](r-2 M)^{m+1} \mathbb{C}[[r]]
$$

Since this is the only condition to be satisfied for poles other than at $r=0, \infty$, without loss of generality, we can take $R=f^{m+1}=\left(\frac{r-2 M}{2 M}\right)^{m+1}$ as a convenient universal multiplier. So that, according to Theorem 2.4 , any rational solution of (23) must satisfy

$$
\delta \in R \mathbb{C}\left[r, r^{-1}\right]=f^{m+1} \mathbb{C}\left[r, r^{-1}\right] .
$$

Finally, we can parametrize any such solution with the following bounded order Laurent polynomial:

$$
\delta=f^{m+1} \sum_{n=\check{n}}^{\hat{n}} d_{n} r^{n}, \quad \text { where } \quad\left\{\begin{array}{l}
\hat{n}=\max \{\hat{m}\} \cup \sigma_{\infty}(e)=\max \{\hat{m}, 0\}, \\
\check{n}=\min \{\check{m}\} \cup \sigma_{0}(e)=\min \left\{\check{m},-s_{0}-s_{1}\right\} .
\end{array}\right.
$$

\subsection{Examples}

We finish with a few explicit examples. Sometimes, in specific examples, a $\delta, \epsilon$ solution for a given $\Delta$ can be found by trial and error. However, when unguided, such a process can be quite laborious. And at the end, if no solution was found, one cannot automatically conclude that a solution does not exist. Using the method presented above, when the trial and error method becomes too laborious, it can be automated using a computer algebra system. Moreover, our method can also furnish a proof that in some situation no solution exists.

Below, we find it helpful to use the notation $\breve{O}\left(r^{p}\right)$ to denote the leading order of a Laurent series at $r=0$ and $\hat{O}\left(r^{q}\right)$ to denote the trailing order of a Laurent series at $r=\infty$.

1. The equation

$$
\mathcal{D}_{0} \circ \delta=\frac{f_{1}}{r^{2}}+\varepsilon \circ \mathcal{D}_{1}
$$

where

$$
\left[\begin{array}{c}
\Delta_{0} \\
\Delta_{1}
\end{array}\right]=\left[\begin{array}{c}
f_{1} \\
0
\end{array}\right]=\left[\begin{array}{c}
\check{O}\left(r^{-1}\right) \\
0
\end{array}\right]=\left[\begin{array}{c}
\hat{O}\left(r^{-1}\right) \\
0
\end{array}\right]
$$

gives rise to the normalized sources

$$
\left|\check{A}_{0}\right|=\left[\begin{array}{c}
0 \\
\infty
\end{array}\right], \quad\left|\check{A}_{2 M}\right|=\left[\begin{array}{c}
0 \\
\infty
\end{array}\right], \quad\left|\hat{A}_{\infty}\right|=\left[\begin{array}{c}
-3 \\
-\infty
\end{array}\right] .
$$

Since $A_{2 M}=T_{2 M}^{-1} \Delta$ has no pole at $r=2 M$, we can choose the universal multiplier to be $R=1 . A_{0}$ and $A_{\infty}$ give the orders $\check{m}=0$ and $\hat{m}=-3$ and hence the Laurent polynomial bounds $\check{n}=\min \{0,-0-1\}=-1$ and $\hat{n}=\max \{-3,0\}$. From this information, we can conclude that there exists the unique solution

$$
\delta=-1, \quad \varepsilon=-1
$$

2. The equation

$$
\mathcal{D}_{0} \circ \delta=\frac{f_{1}}{r^{2}}+\varepsilon \circ \mathcal{D}_{0}
$$

where $\Delta_{0}, \Delta_{1}$ and the corresponding normalized sources are the same as in Example 2. Again, since $A_{2 M}=$ $T_{2 M}^{-1} \Delta$ has no pole at $r=2 M$, we can choose the universal multiplier to be $R=1$. $A_{0}$ and $A_{\infty}$ give the orders $\check{m}=0$ and $\hat{m}=-3$ and hence the Laurent polynomial bounds $\check{n}=\min \{0,-0-0\}=0$ and $\hat{n}=\max \{-3,0\}=0$. From this information, we can conclude that there exists no solution for $\delta, \varepsilon$.

3. The equation

$$
\mathcal{D}_{0} \circ \delta=-\frac{f_{1}}{r^{2}}\left(\mathcal{B}_{l}+\frac{f_{1}}{2}\right)+\varepsilon \circ \mathcal{D}_{0}
$$

where

$$
\left[\begin{array}{c}
\Delta_{0} \\
\Delta_{1}
\end{array}\right]=\left[\begin{array}{c}
-f_{1}\left(\mathcal{B}_{l}+\frac{f_{1}}{2}\right) \\
0
\end{array}\right]=\left[\begin{array}{c}
\check{O}\left(r^{-2}\right) \\
0
\end{array}\right]=\left[\begin{array}{c}
\hat{O}\left(r^{-1}\right) \\
0
\end{array}\right]
$$


gives rise to the normalized sources

$$
\left|\check{A}_{0}\right|=\left[\begin{array}{c}
-1 \\
\infty
\end{array}\right], \quad\left|\check{A}_{2 M}\right|=\left[\begin{array}{c}
0 \\
\infty
\end{array}\right], \quad\left|\hat{A}_{\infty}\right|=\left[\begin{array}{c}
-3 \\
-\infty
\end{array}\right] .
$$

Since $A_{2 M}=T_{2 M}^{-1} \Delta$ has no pole at $r=2 M$, we can choose the universal multiplier to be $R=1 . A_{0}$ and $A_{\infty}$ give the orders $\check{m}=-1$ and $\hat{m}=-3$ and hence the Laurent polynomial bounds $\check{n}=\min \{-1,-0-0\}=-1$ and $\hat{n}=\max \{-3,0\}=0$. From this information, we can conclude that there exists no solution for $\delta, \varepsilon$.

4. The equation

$$
\mathcal{D}_{0} \circ \delta=\Delta+\varepsilon \circ \mathcal{D}_{2}
$$

with

$$
\begin{aligned}
& \Delta= 24 i f_{1} r^{2} \omega^{3}-4 i f\left(6 f f_{1}+6 \mathcal{B}_{l} f_{1}+\mathcal{A}_{l}\right) r \omega \partial_{r} \\
&-2 i\left(\mathcal{A}_{l}+2\left(\mathcal{B}_{l}-3\right)+\left(\mathcal{A}_{l}-\mathcal{B}_{l}\right)\left(1+2 \mathcal{B}_{l}\right)+2\left(\mathcal{A}_{l}+6 \mathcal{B}_{l}\right) f-9 \frac{\mathcal{A}_{l}}{\mathcal{B}_{l}} f^{2}-12 f^{3}\right) \omega \\
&+\frac{f_{1} f \mathcal{B}_{l}\left(-4 f_{1}^{2}+8 f f_{1}-4 \mathcal{B}_{l}+16 f \mathcal{B}_{l}+\mathcal{A}_{l}\right)}{i r \omega} \partial_{r}+\frac{i f_{1} \mathcal{B}_{l}\left(\mathcal{A}_{l}\left(\mathcal{B}_{l}-7 f\right)+12 f\left(1-\left(2+\mathcal{B}_{l}\right) f+f^{2}\right)\right)}{r^{2} \omega} \\
& {\left[\begin{array}{l}
\Delta_{0} \\
\Delta_{1}
\end{array}\right]=\left[\begin{array}{l}
\check{O}\left(r^{-4}\right) \\
\check{O}\left(r^{-4}\right)
\end{array}\right]=\left[\begin{array}{l}
\hat{O}\left(r^{3}\right) \\
\hat{O}\left(r^{2}\right)
\end{array}\right] }
\end{aligned}
$$

gives rise to the normalized sources

$$
\left|\check{A}_{0}\right|=\left[\begin{array}{l}
-3 \\
-3
\end{array}\right], \quad\left|\check{A}_{2 M}\right|=\left[\begin{array}{l}
0 \\
1
\end{array}\right], \quad\left|\hat{A}_{\infty}\right|=\left[\begin{array}{l}
1 \\
2
\end{array}\right] .
$$

Since $A_{2 M}=T_{2 M}^{-1} \Delta$ has no pole at $r=2 M$, we can choose the universal multiplier to be $R=1 . A_{0}$ and $A_{\infty}$ give the orders $\check{m}=-3$ and $\hat{m}=2$ and hence the Laurent polynomial bounds $\check{n}=\min \{-3,-0-2\}=-3$ and $\hat{n}=\max \{2,0\}=2$. From this information, we can conclude that there exists a unique solution

$$
\begin{aligned}
\delta= & -6 i f_{1} f r^{3} \omega \partial_{r}-i\left(6 f f_{1}+12 \mathcal{B}_{l} f_{1}+\mathcal{A}_{l}\right) r^{2} \omega+\frac{i f \mathcal{B}_{l}\left(4 \mathcal{A}_{l}-4-2 f_{1}+24 f f_{1}+3 \mathcal{B}_{l}+f l(l-1)\right) r \partial_{r}}{2 \omega} \\
& +\frac{i\left(\mathcal{A}_{l}^{2}+2 \mathcal{A}_{l}\left(9+5 \mathcal{B}_{l}\right) f-6 \mathcal{B}_{l} f^{2}\left(-8 f_{1}-6+3 \mathcal{B}_{l}\right)\right)}{4 \omega},
\end{aligned}
$$

with $\varepsilon$ given by (22). The above result was obtained and checked with computer algebra.

5. The equation

$$
\mathcal{D}_{1} \circ \delta=\Delta+\varepsilon \circ \mathcal{D}_{2},
$$

with

$$
\begin{gathered}
\Delta=-24 i f_{1} f r \omega \partial_{r}-4 i \mathcal{A}_{l} \omega+\frac{6 f_{1} f(3 f-1) \mathcal{B}_{l}}{i r \omega} \partial_{r}-\frac{-i f_{1} \mathcal{B}_{l}\left(18 f f_{1}-6 f \mathcal{B}_{l}+\mathcal{A}_{l}\right)}{r^{2} \omega} \\
{\left[\begin{array}{l}
\Delta_{0} \\
\Delta_{1}
\end{array}\right]=\left[\begin{array}{l}
\check{O}\left(r^{-3}\right) \\
\check{O}\left(r^{-3}\right)
\end{array}\right]=\left[\begin{array}{l}
\hat{O}\left(r^{2}\right) \\
\hat{O}\left(r^{1}\right)
\end{array}\right]}
\end{gathered}
$$

gives rise to the normalized sources

$$
\left|\check{A}_{0}\right|=\left[\begin{array}{l}
-2 \\
-2
\end{array}\right], \quad\left|\check{A}_{2 M}\right|=\left[\begin{array}{l}
0 \\
1
\end{array}\right], \quad\left|\hat{A}_{\infty}\right|=\left[\begin{array}{l}
0 \\
1
\end{array}\right] .
$$

Since $A_{2 M}=T_{2 M}^{-1} \Delta$ has no pole at $r=2 M$, we can choose the universal multiplier to be $R=1 . A_{0}$ and $A_{\infty}$ give the orders $\check{m}=-2$ and $\hat{m}=1$ and hence the Laurent polynomial bounds $\check{n}=\min \{-2,-1-2\}=-3$ and $\hat{n}=\max \{1,0\}=1$. From this information, we can conclude that there exists a unique solution

$$
\delta=-12 i f_{1} r^{2} \omega+\frac{2 i f\left(\mathcal{B}_{l}-2 f_{1}\right)\left(\mathcal{B}_{l}-2 f+f_{1}\right) r \partial_{r}}{\omega}+\frac{i\left(\frac{\mathcal{A}_{l}^{2}}{\mathcal{B}_{l}}+6 \frac{\mathcal{A}_{l}}{\mathcal{B}_{l}}\left(\mathcal{B}_{l}+3\right) f-18\left(\mathcal{B}_{l}-4\right) f^{2}-36 f\right)}{3 \omega},
$$

with $\varepsilon$ given by (22). The above result was obtained and checked with computer algebra. 
The solution from Example 1 can be generalized to

$$
\mathcal{D}_{s_{0}} \frac{1}{\left(s_{0}^{2}-s_{1}^{2}\right)}=\frac{f_{1}}{r^{2}}+\frac{1}{\left(s_{0}^{2}-s_{1}^{2}\right)} \mathcal{D}_{s_{1}},
$$

which actually works for any complex values of $s_{0}, s_{1}$, except for $s_{0}= \pm s_{1}$. We obtained this parametric solution by trial and error, while trying to understand and generalize some identities from [6]. However, simply having this formula does not tell us whether it is the unique solution. Applying our systematic approach, we can check uniqueness as we did in Example 1 for $s_{0}=0$ and $s_{1}=1$, but only for specific values of $s_{0}, s_{1}$ at a time. The reason is that the lower bound $\check{n}$ on the Laurent polynomial order of $\delta$ in $(30)$ is influenced by $\min \sigma_{0}(e)=-s_{0}-s_{1}($ at least for non-negative integer values of the $s_{i}$ ), which depend on the $s_{i}$.

Note also that the above formula is singular for $s_{0}= \pm s_{1}$ and no longer tells us anything about the existence of solutions in those cases. On the other hand, our systematic approach can check that indeed no solution exists, again on a case by case basis, as we did for $s_{0}=0$ in Example 2.

In Equation (88) of [7], we had managed to reduce a $3 \times 3$ upper triangular system with Regge-Wheeler operators on the diagonal and a single rational non-vanishing off-diagonal component. Incidentally, the solution from Example 1 was instrumental in that simplification. The existence of a solution in Example 3 would mean that this system could be further reduced to diagonal form by a rational transformation. This question was left open in [7]. However, the non-existence of such solutions, as we have just confirmed in Example 1, proves that no such further simplification is possible.

In [7], we have arrived at an upper-triangular Regge-Wheeler system as a partial decoupling of the radial mode equations of the vector wave equation (which could be interpreted as the harmonic or Lorenz gauge-fixed version of Maxwell's equations) on the background of a Schwarzschild black hole. That work was strongly inspired by [6], which achieved a similar decoupling for the Lichnerowicz equation (which could be interpreted as the harmonic or de Donder gauge-fixed version of linearized Einstein's equations) also on Schwarzschild. The methods and results result achieved in [6] are unfortunately somewhat obscure and implicit. We aim to clarify those results using the systematic methods that we have outlined in [7] and in the present work. For instance, the existence of the solutions from Examples 4 and 5 is equivalent to Equations (3.49-51) from [6], which were instrumental to their main decoupling results, but apparently obtained by trail and error, without a clear guide to how they could be reproduced independently. Fortunately, Examples 4 and 5 show that our systematic approach can rediscover these formulas in a straight forward way using computer algebra.

\section{Discussion}

The main goal of this work was to conclusively decide when it is or is not possible to reduce an upper triangular rational ODE system like (18) to diagonal form by a transformation like (14) with rational coefficients, where on the diagonal we have generalized Regge-Wheeler operators. This question was left open in our previous work [7]. In Section 3, we showed how to reduce this question to the existence of a rational solution to an auxiliary rational ODE system. In Section 2 we showed that, under mild hypotheses, the existence of a rational solution of a rational ODE system can be reduced to a finite dimensional linear algebra problem. Hence, such a question can always be conclusively decided, at least on a case by case basis. In Section 4.1, we gave several examples illustrating our methods. These examples reproduce, in a systematic way, some identities previously discovered by voluminous trial and error calculations in [6].

These identities were used in [7] to significantly simplify, after a separation of variables, the coupled radial mode equations of the vector wave equation on Schwarzschild spacetime. Our Example 3 shows that this simplification cannot be further improved. The vector wave equation plays a role relative to the Maxwell equation that is analogous to the Lichnerowicz equation relative to the linearized Einstein equations. In a future work, we will further build on the results of [6] to apply to the Lichnerowicz equation the same simplifications as were applied to the vector wave equation in [7]. The methods developed in this work, will help decide how much these simplifications could be improved. Of course, it will also be very interesting to see how much the simplifications studied jointly in [7] and the current work will translate from the (non-rotating) Schwarzschild black hole to the significantly more complicated case of the (rotating) Kerr black hole.

An interesting generalization of the question of the existence of rational solutions to the rational ODE $e[u]=v$ is the characterization of the image of $e$ when applied to arbitrary rational arguments. An equivalent question is the characterization of the rational cokernel of $e$. Then, even if no rational solution to $e[u]=v$ exists, precisely identifying the equivalence class of $v$ in the cokernel of $e$ might allow us to choose a representative from the equivalence class of $v$ that is simplest, with respect to some reasonable criteria. Such questions also have connections 
with the theory of $\mathcal{D}$-modules with rational coefficients [10, Ch.2], [9, Sec.10.5], which is an algebraic formalism for studying linear differential equations, especially those with polynomial or rational coefficients. These topics may also be explored in future work.

\section{Acknowledgments}

Research of the author was partially supported by the GAČR project 18-07776S and RVO: 67985840. The author also thanks Francesco Bussola for help with converting Equations (3.49-51) of [6] into the form given in Examples 4 and 5 .

\section{References}

[1] S. Abramov, "Rational solutions of linear differential and difference equations with polynomial coefficients," USSR Computational Mathematics and Mathematical Physics 29 (1989) 7-12. http://mi . mathnet.ru/zvmmf3350.

[2] S. A. Abramov, "Problems in computer algebra that are connected with a search for polynomial solutions of linear differential and difference equations," Vestnik Moskovskogo Universiteta. Seriya XV. Vychislitel'naya Matematika i Kibernetika no. 3, (1989) 56-60.

[3] S. A. Abramov, Elements of the computer algebra of linear ordinary differential, difference and q-difference operators. MCCME, Moscow, 2014. In Russian.

[4] S. A. Abramov, "Search of rational solutions to differential and difference systems by means of formal series," Programming and Computer Software 41 (2015) 65-73.

[5] S. A. Abramov, M. Bronstein, and M. Petkovšek, "On polynomial solutions of linear operator equations," in Proceedings of the 1995 international symposium on Symbolic and algebraic computation - ISSAC '95, pp. 290-296. ACM Press, New York, 1995.

[6] M. V. Berndtson, Harmonic gauge perturbations of the Schwarzschild metric. PhD thesis, University of Colorado, 2007. arXiv:0904.0033.

[7] I. Khavkine, "Explicit triangular decoupling of the separated vector wave equation on Schwarzschild into scalar Regge-Wheeler equations," 2017. arXiv:1711.00585.

[8] J. G. Rosa and S. R. Dolan, "Massive vector fields on the Schwarzschild spacetime: quasinormal modes and bound states," Physical Review D 85 (2012) 044043, arXiv:1110.4494.

[9] W. M. Seiler, Involution: The Formal Theory of Differential Equations and its Applications in Computer Algebra, vol. 24 of Algorithms and Computation in Mathematics. Springer, Berlin, 2010.

[10] M. van der Put and M. F. Singer, Galois Theory of Linear Differential Equations, vol. 328 of Grundlehren der mathematischen Wissenschaften. Springer, Berlin, 2003.

[11] W. Wasow, Asymptotic Expansions for Ordinary Differential Equations, vol. XIV of Pure and Applied Mathematics. Interscience, New York, NY, 1965. 